\title{
Neural fractalkine expression is closely linked to pain and pancreatic neuritis in human chronic pancreatitis
}

\author{
Güralp O Ceyhan ${ }^{1,2}$, Stefanie Deucker ${ }^{2}$, Ihsan Ekin Demir ${ }^{1}$, Mert Erkan ${ }^{1}$, Martin Schmelz ${ }^{3}$, Frank Bergmann ${ }^{4}$, \\ Michael W Müller ${ }^{1}$, Thomas Giese ${ }^{5}$, Markus W Büchler ${ }^{2}$, Nathalia A Giese ${ }^{2}$ and Helmut Friess ${ }^{1}$
}

The chemokine fractalkine induces migration of inflammatory cells into inflamed tissues, thereby aggravating inflammatory tissue damage and fibrosis. Furthermore, fractalkine increases neuropathic pain through glial activation, which can be diminished by blocking of its receptor, $C X 3 C R 1$, through neutralizing antibodies. As chronic pancreatitis (CP) is characterized by tissue infiltration of inflammatory cells, fibrosis, pancreatic neuritis and severe pain, the roles of fractalkine and CX3CR1 were investigated in CP $(n=61)$ and normal pancreas (NP, $n=21)$ by QRT-PCR, western blot and immunohistochemistry analyses. Their expression correlated with the severity of pancreatic neuritis, fibrosis, intrapancreatic nerve fiber density and hypertrophy, pain, CP duration and with the amount of inflammatory cell infiltrate immuno-positive for CD45 and CD68. To investigate the influence of fractalkine on pancreatic fibrogenesis, human pancreatic stellate cells (hPSCs) were isolated from patients with CP, incubated with fractalkine and then Collagen-1 and $\alpha$-smooth muscle actin ( $\alpha$-SMA) expressions were measured. CX3CR1, but not fractalkine, mRNA was overexpressed in CP. In contrast, the protein levels of both $C X 3 C R 1$ and fractalkine were upregulated. Neuro-immunoreactivity for fractalkine and $C X 3 C R 1$ was strongest in patients suffering from severe pain and pancreatic neuritis. Long-term suffering from CP was noticeably related to increased neural immunoreactivity of fractalkine. Furthermore, fractalkine and CX3CR1 mRNA overexpressions were associated with enhanced lymphocyte and macrophage infiltration. Advanced fibrosis was associated with increased fractalkine expression, whereas in vitro fractalkine had no significant impact on collagen-1 and $\alpha$-SMA expressions in hPSCs. Therefore, pancreatic fractalkine expression appears to be linked to visceral pain and to the recruitment of inflammatory cells into the pancreatic tissue and nerve fibers, with subsequent pancreatic neuritis. However, pancreatic fibrogenesis is probably indirectly influenced by fractalkine. Taken together, these novel findings suggest that $C X 3 C R 1$ represents a potential novel therapeutic target to reduce inflammation and modulate pain in CP. Laboratory Investigation (2009) 89, 347-361; doi:10.1038/labinvest.2008.170; published online 19 January 2009

KEYWORDS: fractalkine; CX3CR1; chronic pancreatitis; pain; pancreatic neuritis; fibrosis

Chronic pancreatitis (CP) is an inflammatory disease characterized by irreversible and progressive destruction of the pancreas, resulting in severe exocrine and endocrine insufficiency. ${ }^{1,2}$ The destruction of the pancreatic parenchyma is accompanied by severe fibrosis with distinct accumulation of extracellular matrix (ECM) and impressive infiltration of various subsets of inflammatory cells. ${ }^{1,3}$ There is a growing body of evidence that mononuclear cells modulate fibrogenesis by supporting the activation of human pancreatic stellate cells (hPSCs), which are the major source of intrapancreatic ECM production. ${ }^{4-7}$ Once activated,
hPSCs not only release ECM proteins but also produce proinflammatory cytokines and chemokines. ${ }^{4-7}$

Another characteristic inflammatory event in $\mathrm{CP}$ is represented by perineural mononuclear cell infiltration. The layers of the perineurium are altered and damaged, thus they no longer provide a protective barrier. At the sites of inflammation, mononuclear cells can easily infiltrate the damaged perineurium and cause the characteristic pancreatic neuritis, which is strongly associated with abdominal pain in $\mathrm{CP}^{8-10}$ However, the precise pathophysiology of the neuro-immune crosstalk, and of pain initiation and maintenance in CP remains unclear.

\footnotetext{
${ }^{1}$ Department of Surgery, Technische Universität München, Munich, Germany; ${ }^{2}$ Department of General Surgery, University of Heidelberg, Heidelberg, Germany; ${ }^{3}$ Institute for Anaesthesiology Mannheim, University of Heidelberg, Mannheim, Germany; ${ }^{4}$ Institute of Pathology, University of Heidelberg, Heidelberg, Germany and ${ }^{5}$ Institute for Immunology, University of Heidelberg, Heidelberg, Germany

Correspondence: Dr GO Ceyhan, MD, Department of Surgery, Klinikum Rechts der Isar, Technische Universität München, Ismaninger Str. 22, D-81675 Munich, Germany. E-mail: ceyhan@chir.med.tu-muenchen.de

Received 07 August 2008; revised 15 October 2008; accepted 29 October 2008
} 
At the sites of injury and inflammation, chemokines and adhesion molecules provide signals for leukocyte trafficking, including leukocyte adhesion and migration. Fractalkine (CX3CL1), the unique member of the fourth class of chemokines $(C X 3 C)$, is expressed on endothelial cells and lymphocytes, and is the only other known chemokine to be expressed on spinal neurons and sensory afferents, other than monocyte chemoattractant protein-1 (MCP-1). ${ }^{11-15}$ The highly selective fractalkine receptor CX3CR1 is expressed on cytotoxic effector lymphocytes, including natural killer cells, cytotoxic T cells and macrophages. ${ }^{16}$ In the nervous system, CX3CR1 is expressed in microglia in the spinal cord, by satellite cells in the dorsal root ganglia (DRG) and in human embryonic neurons. ${ }^{14,17}$ A distinctive feature of fractalkine is that it exhibits properties of both traditional chemokines and adhesion molecules. As a chemoattractant, fractalkine induces the migration and extravasation of natural killer cells, cytotoxic lymphocytes and macrophages into inflamed tissues and thereby aggravating tissue damage. ${ }^{11,18}$ Moreover, by acting as an adhesion molecule, fractalkine can capture and enhance the migration of CX3CR1-expressing mononuclear cells through direct cell-to-cell interactions. ${ }^{19,20}$ Recent data show that fractalkine and CX3CR1 are involved in fibrogenesis, and are associated with renal and liver fibrosis. ${ }^{21,22}$

Independently from its peripheral pro-inflammatory effects, peripheral nerve damage leads to up-regulation of fractalkine in DRG and consequently to neuropathic pain, with activation of $C X 3 C R 1$ on spinal microglia being a crucial mechanism for neuronal sensitization. ${ }^{12,14,23,24}$ However, also peripherally present are inflammatory mediators such as $T N F-\alpha$ and $I L-1$, which might contribute to neuropathic pain, if given access to the peripheral nerve through intraneural injection ${ }^{25}$ or peri-sciatic-zymosan-induced inflammation. ${ }^{26}$ In contrast, intraneural injection of fractalkine into the sciatic nerve was recently shown to attenuate neuropathic pain-related behavior in a spared nerve injury model of neuropathic pain in mice. ${ }^{27}$

In summary, fractalkine has pro-inflammatory, painmodulating and fibrogenic effects. As all these three factors, that is inflammation, pain and fibrosis, determine the prognosis of $\mathrm{CP}$ patients and often necessitate surgical intervention, we studied fractalkine expression and its relation to inflammation, pain and fibrosis in the chronically inflamed pancreas.

\section{MATERIALS AND METHODS} Patients and Tissues

A total of $61 \mathrm{CP}$ tissue samples were collected from patients undergoing pancreatic head resection ( 45 males, age $46 \pm 2$ years; 16 females, age $42 \pm 3$ years). The etiology of the pancreatitis was alcoholic CP $(n=26)$, biliary CP $(n=5)$, idiopathic $\mathrm{CP}(n=24)$ and autoimmune pancreatitis $(n=6)$. Written informed consent was obtained from all patients. Normal pancreatic tissue samples (NP) were collected from organ donors whenever there was no appropriate recipient for transplantation $(n=21 ; 13$ males, 8 females; median age 40 years). During organ procurement, the pancreas, like the other organs (kidney, liver) used for solid organ transplantation, was perfused with a University of Wisconsin solution (UW) or with histidine-tryptophan-ketoglutarate solution. Only pancreata that were explanted in our department were taken for further research studies. The pancreata were removed and transported in the same conservation solution on ice under sterile conditions after tissue preservation. Therefore, the median time from cross-clamp to processing was approximately about $30 \mathrm{~min}$. This study was approved by the Ethics Committee of the University of Heidelberg (Heidelberg, Germany).

Resected pancreatic tissues were divided into several parts and the aliquots were (a) frozen in liquid nitrogen and stored at $-80^{\circ} \mathrm{C}$ for protein extraction, (b) taken into RNAlater (Ambion, Huntington, UK) for RNA extraction, (c) fixed in $5 \%$ paraformaldehyde, and later embedded in paraffin for immunohistochemical analysis and (d) immediately processed for hPSC isolation.

\section{Reagents}

The following reagents were purchased: RPMI-1640, DMEM, Trypsin-EDTA and penicillin-streptomycin from Invitrogen (Karlsruhe, Germany); fetal calf serum (FCS) from PAN Biotech (Aidenbach, Germany); fractalkine and CX3CR1 rabbit polyclonal antibodies from Abcam (Cambridge, UK); Collagen-1 goat polyclonal antibody from Santa Cruz Biotechnology (Heidelberg, Germany); CD45, CD68 and $\alpha-S M A$ mouse monoclonal and PGP9.5 rabbit polyclonal antibody from DAKO Cytomation (Hamburg, Germany) and $\gamma$-tubulin goat polyclonal antibody from Santa Cruz Biotechnology. The DAKO Envision system was used for immunohistochemistry and the ECL immunoblotting detection reagents from Amersham Biosciences (Buckinghamshire, UK) were used for western blot analysis. Recombinant human fractalkine was purchased from R\&D Systems (Wiesbaden, Germany) and TNF- $\alpha$ from Chemicon (Temecula, CA, USA). All other chemicals were from Sigma Chemical Company (Taufkirchen, Germany).

\section{Real-Time Light Cycler ${ }^{\circledR}$ Quantitative PCR}

Extraction of mRNA from normal and $\mathrm{CP}$ tissues and of hPSCs was carried out by automated isolation using the MagNA Pure LC instrument and isolation kits I (for cells) and II (for tissue) (Roche Applied Science, Mannheim, Germany). cDNA was prepared using the first-strand cDNA synthesis kit for RT-PCR (Roche Applied Science). Quantitative real-time (PCR) QRT-PCR was carried out with the Light Cycler Fast Start DNA SYBR Green kit. Primers for fractalkine, CX3CR1 and MCP-1 were obtained from Search-LC (Heidelberg, Germany). The calculated number of specific transcripts was normalized to the housekeeping genes, cyclophilin B and hypoxanthine guanine phospho-ribosyltransferase, 
and expressed as amount per microliter of input CDNA, as described earlier. ${ }^{28}$

\section{Western Blot Analysis}

Protein extraction and western blot analysis of cell culture monolayers or tissues were carried out as described earlier. ${ }^{29}$ A total of $20 \mu \mathrm{g}$ of protein/well in the tissue and cell lysates and $15 \mu \mathrm{g}$ in the supernatants were separated and electroblotted, and the membrane was exposed to fractalkine and CX3CR1 antibodies (dilution of 1:2500 and 1:1000) at $4{ }^{\circ} \mathrm{C}$ overnight. Signal detection was performed using an enhanced chemiluminescence reaction. After film scanning, densitometric analysis was carried out using the ImageJ software provided by the National Institutes of Health. Specific signal intensity was calculated and was corrected for the matching equal loading densities. ${ }^{30}$ To ensure the specificity of the fractalkine antibody, HUVECs were stimulated with $10 \mathrm{ng} / \mathrm{ml}$ $T N F-\alpha$ for $12 \mathrm{~h}$. Equal loading was assured by stripping the blots and re-probing with anti- $\gamma$-tubulin (1:5000).

\section{Immunohistochemistry}

Paraffin-embedded tissue sections of normal pancreas and $\mathrm{CP}$ samples were analyzed by immunostaining, using the DAKO Envision system with DAB as chromogene, as described earlier. ${ }^{29} P G P 9.5$, fractalkine and $C X 3 C R 1$ antibodies were used at a dilution of 1:1000, 1:300 and 1:500, respectively. Standardized color reaction time was used for fractalkine $(1.5 \mathrm{~min})$ and CX3CR1 (45 s) in all consecutive slides. Non-immunized rabbit IgG (DAKO) was used as a negative control. Digital imaging was performed using the Zeiss AxioCam HR system (Carl Zeiss AG, Oberkochen, Germany).

\section{Clinicopathological Analysis}

Consecutive sections obtained from the pancreatic tissue of each patient were stained with hematoxylin and eosin for concomitant histomorphological examination. Histopathological analysis was carried out by two independent observers blinded to QRT-PCR, pain, CD45 and CD68 data, followed by resolution of any differences by joint review and consultation with a third observer. Histomorphological evaluation of the specimens included the severity of pancreatic neuritis and the severity of fibrosis, as previously earlier. ${ }^{31}$ The severity of pancreatic neuritis was scored as absent (0), perineural (score I) or endoneural (score II). The severity of fibrosis was graded according to a scoring system (Table 1). The severity of fibrosis was revealed by the addition of intralobular and perilobular fibrosis scores as mild $0(0-4)$, moderate I (5-9) or severe II (10-12) fibrosis.

No pathomorphological abnormalities were detected within the group of normal pancreas.

\section{Neural Immunoreactivity}

Analysis of the fractalkine and CX3CR1 neuroimmunoreactivity in intrapancreatic nerves was performed by an independent observer (MWM) blinded to patient diagnosis, disease etiology, histopathological changes and pain history. From each section, all stained and unstained visible nerves were identified, using a consecutive PGP9.5-immunostained section of the same tissue as reference. All intrapancreatic nerves on each tissue section were analyzed for fractalkine and CX3CR1 immunoreactivity. The evaluation included the intensity (none $=0$, mild $=1$, moderate $=2$ or strong $=3$ ) and the area of neural immunoreactivity $(<30 \%=1, \quad 30-60 \%=2$ or $>60 \%=3)$ for fractalkine and CX3CR1. To calculate the neural immunoreactivity score, intensity and area of immunoreactivity were multiplied. The median of all analyzed nerves was set as the representative score for fractalkine and CX3CR1 neuroimmunoreactivity of every single patient.

\section{Quantitative Analysis of Nerve Number and Total Nerve Area in CP}

Intrapancreatic nerves in tissue sections of normal pancreas and CP patients were immunolabeled using the pan-neuronal marker PGP9.5. To quantify the total number and the total nerve area, the entire pancreatic tissue sections were scanned at high magnification $(\times 100)$ and reconstructed into a digital mosaic using the Zeiss KS300 program, with analysis of all PGP9.5-immunoreactive nerves as shown before. ${ }^{31}$

\section{Pain Scoring and Disease Duration}

In all CP patients, the individual pain score was registered before operation, including pain intensity and frequency as described earlier. ${ }^{31}$ The intensity of pain was graded by using the scale as follows: $0=$ none, $1=$ mild, $2=$ moderate and $3=$ strong pain. The frequency of pain was graded as $1=$ monthly, $2=$ weekly and $3=$ daily. To calculate the pain severity, pain intensity and frequency were multiplied. According to the final pain score, the patients were divided into three groups, as described earlier: ${ }^{31,32}$ Pain 0 (0), representing the group of patients who did not have any pain; Pain I (1-3), representing the group of patients who suffered from mild pain; and Pain II (4-9), the group of patients with moderate-to-severe pain. Accordingly, patients' individual time period of disease $(\mathrm{CP})$ was asked. The patients' disease duration was recorded in weeks.

\section{Quantitative Analysis of CD45 and CD68 Immunoreactivity in CP}

To quantify the intensity of inflammatory cell and macrophage infiltration, tissue sections of $\mathrm{CP}$ patients were immunostained using the pan-leukocyte markers $C D 45$ and CD68 for macrophages. Subsequently, the entire pancreatic tissue sections were scanned and reconstructed into a mosaic image using the Zeiss KS300 program. The inflammatory cells, immuno-labeled for CD45 and CD68, were automatically detected and evaluated by the KS300 software in the scanned tissue sections. The intensity of CD45 and CD68 was expressed as the percentage of area occupied by 
immunoreactive cells within the total area of the analyzed section.

\section{Isolation of hPSCs}

hPSCs were isolated from freshly resected pancreatic tissue of $\mathrm{CP}$ patients using the outgrowth method described by Bachem et al. ${ }^{5}$ HPSCs were cultivated in a 1:1 dilution of Ham's F-12 nutrient medium and low-glucose DMEM supplemented with penicillin, streptomycin, amphotericin and $20 \%$ of FCS, as described earlier. ${ }^{5}$

\section{Fractalkine stimulation of hPSCs}

HPSCs were grown in six-well plates for protein and RNA extraction. When the cells reached $70 \%$ confluence, fresh medium was supplemented with $1 \%$ of FCS containing fractalkine (10 and $100 \mathrm{ng} / \mathrm{ml})$. PBS was used as a control. Changes in Collagen- 1 and $\alpha$-SMA mRNA expressions in

Table 1 Scoring system for the evaluation of fibrosis in chronic pancreatitis

\begin{tabular}{lllll}
\hline & \multicolumn{4}{c}{ Severity of fibrosis } \\
\cline { 2 - 5 } & Mild & Moderate & Severe & Score \\
\hline $\begin{array}{l}\text { Perilobular } \\
\text { Focal }\end{array}$ & 1 & 2 & 3 & $1-3$ \\
Diffuse & 4 & 5 & 6 & $4-6$ \\
& & & & \\
Intralobular & & & & $1-3$ \\
Focal & 1 & 2 & 3 & $4-6$ \\
Diffuse & 4 & 5 & 6 & \\
\hline
\end{tabular}

hPSCs were analyzed in cell lysates after 12, 24 and $48 \mathrm{~h}$ using QRT-PCR. After an incubation time of $72 \mathrm{~h}$, western blot analyses of $\alpha$-SMA (1:5000) and Collagen-1 (1:1000) were performed in the hPSC lysates. Equal loading was assured by stripping the blots and re-probing with anti- $\gamma$-tubulin (1:5000). Furthermore, the secreted Collagen-1 (1:1000) was analyzed in the hPSC supernatants, by western blot analysis, as shown earlier. ${ }^{30}$ For this, 20000 hPSCs were placed per well after a 24-h stimulation with fractalkine.

All experiments were repeated thrice.

\section{Statistical Analysis}

Statistical analysis was carried out using the GraphPad Prism 4 Software. The Shapiro-Wilk test was used to evaluate data distribution. Results are expressed as mean \pm s.e.m. The Mann-Whitney $U$-test was carried out in the comparisons of intrapancreatic fractalkine and CX3CR1 mRNA expressions in CP samples with normal control patients. For multiple comparisons, analysis of variance for random measures followed by Bonferroni's post hoc test was used. The relationship between fractalkine and CX3CR1 mRNA expression/neural immunoreactivity with severity pancreatic neuritis, fibrosis, pancreatic neuropathy, duration of CP-disease and CD45/ CD68 immunoreactivities were examined using the Spearman's $\rho$-test. Two-sided $P$-values were always computed, and an effect was considered statistically significant at a $P$-value $\leq 0.05$.

\section{RESULTS}

Pathomorphological Features and Pain Related to CP etiology

Among the four subtypes of CP (alcoholic, biliary, hereditary and autoimmune), the features of inflammatory cell infiltration (CD45 and CD68) and neural hypertrophy were

Table 2 Relationship between the etiology of chronic pancreatitis and mRNA expressions of fractalkine and CX3CR1

\begin{tabular}{|c|c|c|c|c|c|}
\hline & Alcoholic CP $(n=26)$ & Biliary CP $(n=5)$ & Idiopathic CP $(n=24)$ & $\operatorname{AIP}(n=6)$ & $P$-value \\
\hline Fibrosis score & $9.2 \pm 0.6$ & $3.2 \pm 2.0$ & $7.6 \pm 0.8$ & $7.5 \pm 2.0$ & $=0.022$ \\
\hline CD68 & $0.4 \pm 0.06$ & $0.1 \pm 0.05$ & $0.7 \pm 0.4$ & $1.1 \pm 0.4$ & $=0.49$ \\
\hline Neural hypertrophy ${ }^{a}$ & $5725 \pm 1577$ & $586 \pm 356$ & $7794 \pm 2442$ & $1637 \pm 572$ & $=0.35$ \\
\hline Neuritis score & $1.6 \pm 0.1$ & $0.4 \pm 0.2$ & $1.2 \pm 0.1$ & $1.0 \pm 0.4$ & $=0.016$ \\
\hline Pain score & $6.0 \pm 0.7$ & $1.2 \pm 1.2$ & $4.6 \pm 0.7$ & $3.0 \pm 1.3$ & $=0.031$ \\
\hline Fractalkine ${ }^{c}$ & $228 \pm 41$ & $131 \pm 37$ & $313 \pm 53$ & $222 \pm 39$ & $=0.31$ \\
\hline$C \times 3 C R 1^{C}$ & $44 \pm 7$ & $26 \pm 13$ & $45 \pm 12$ & $100 \pm 20$ & $=0.07$ \\
\hline
\end{tabular}

AIP, autoimmune pancreatitis; $C P$, chronic pancreatitis.

${ }^{\mathrm{a}}$ Expressed as $\mu \mathrm{m}^{2}$.

${ }^{\mathrm{b}}$ Nerve number in $1 \mathrm{~mm}^{2}$ tissue section.

Cexpressed as mRNA transcripts/ $\mu \mathrm{l}$. 
not significantly associated with CP etiology (Table 2). Neural hypertrophy tended to be highest in hereditary and alcoholic CP, but did not reach statistical significance, whereas increased neurite density was uppermost in patients with hereditary and alcoholic CP (Table 2). In patients with biliary $\mathrm{CP}$, the severity of fibrosis was not as pronounced as in those with hereditary, autoimmune or especially alcoholic CP. Similar discrepancies were observed regarding pain severity, with highest pain scores determined in patients with alcoholic CP (Table 2).

\section{Expression of Fractalkine and CX3CR1 in CP}

Quantitative RT-PCR analysis was carried out to investigate fractalkine and CX3CR1 mRNA expressions in NP and $\mathrm{CP}$ tissues. Fractalkine mRNA level of $279 \pm 31$ transcripts/ $\mu \mathrm{l}$ (mean \pm s.e.m.) did not differ from that of NP, with $238 \pm 27$ transcripts/ $\mu$ l (Figure 1a). In contrast, the CX3CR1 mRNA level increased significantly $(P<0.0001)$ up to nearly threefold in CP $(48 \pm 7)$ compared with NP $(13 \pm 2$; Figure $1 b)$. Neither fractalkine nor CX3CR1 mRNA expression was significantly altered among alcoholic, biliary, hereditary or autoimmune CP (Table 2). Western blot analysis revealed a remarkable increase for both fractalkine (8.0-fold) and CX3CR1 (6.9-fold) proteins in CP compared with NP (Figure $1 \mathrm{c}$ and $\mathrm{d}$ ). Sensitivity of the fractalkine antibody was ensured indicating upregulated fractalkine protein in HUVECs when stimulated with TNF- $\alpha$ (Figure 1e).

\section{Immunolocalization of Fractalkine and CX3CR1}

To define the localization of fractalkine and CX3CR1 in CP and the normal pancreas, immunohistochemical analysis was carried out. In the normal pancreas, fractalkine showed a weak immunoreactivity in endothelial cells and moderate immunoreactivity in islets (Figure 2a and c). CX3CR1 was detected only in inflammatory cells (Figure $2 \mathrm{~g}-\mathrm{i}$, Table 3 ). In $\mathrm{CP}$, the endothelial cells showed moderate fractalkine immunoreactivity (Figure 2d), which was also identified in tubular complexes and ductal cells, and strong immunoreactivity was identified in islets (Figure $2 \mathrm{f}$, Table 3 ). More prominent changes were observed for CX3CR1 in CP, with strong immunoreactivity in the smooth muscle of arteries, in tubular complexes and inflammatory cells (Figure 2j-1, Table 3). Regarding the acini in CP, both fractalkine and CX3CR1 showed weak-to-moderate
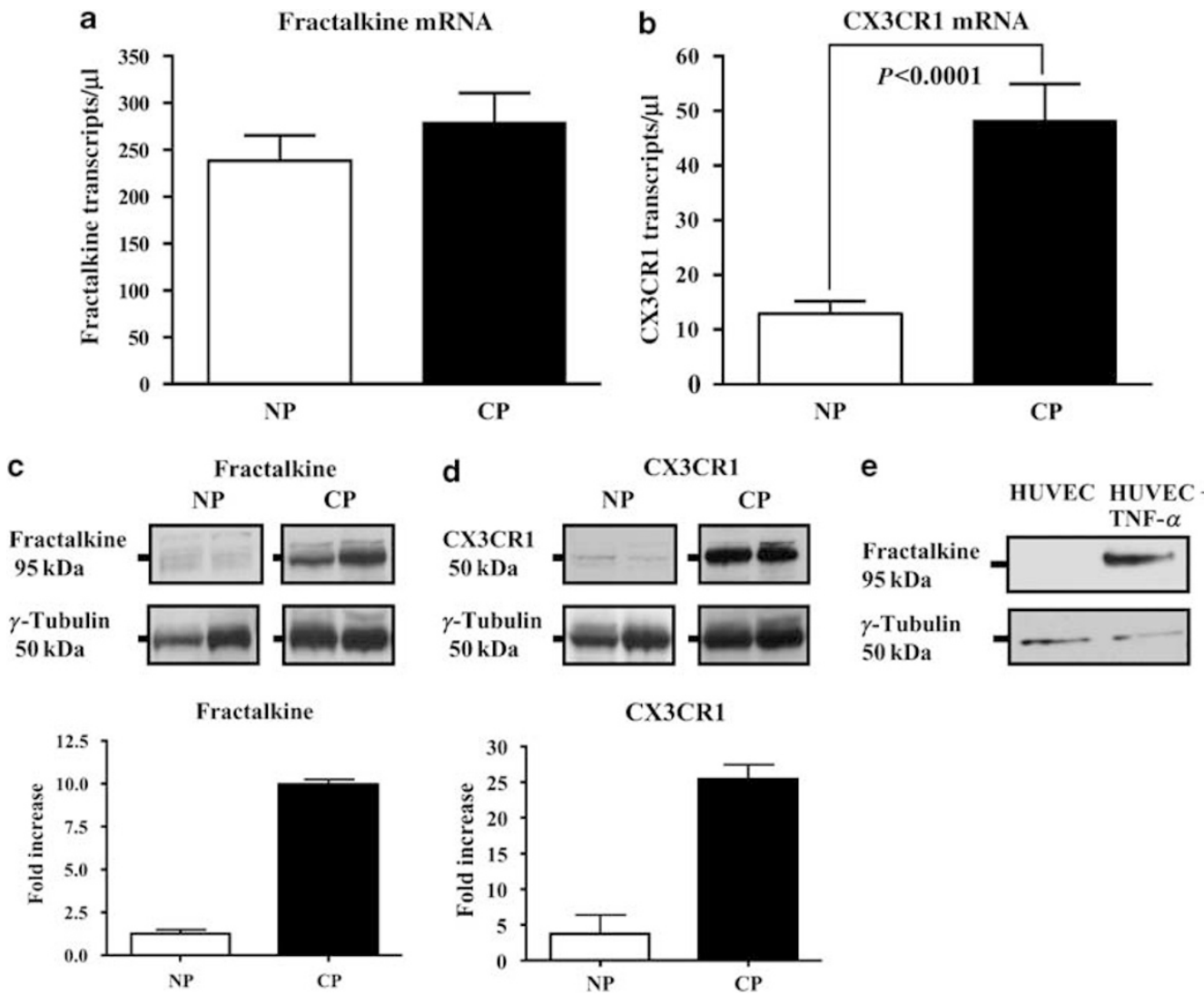

Figure 1 Quantitative RT-PCR analysis of fractalkine (a) and CX3CR1 (b) in pancreatic tissue sections from patients with normal pancreas (NP) and chronic pancreatitis (CP). Western blot analysis of fractalkine (c) and CX3CR1 (d) in NP and CP, showing an 8.0- and 6.9-fold increase, respectively, in CP compared with normal pancreas. (e) The sensitivity of fractalkine was ensured by the stimulation of HUVECs with $10 \mathrm{ng} / \mathrm{ml}$ of $T N F-\alpha$, which is known to upregulate fractalkine. Equal protein loading is verified with $\gamma$-tubulin. Data are presented as mean \pm s.e.m. 
Fractalkine
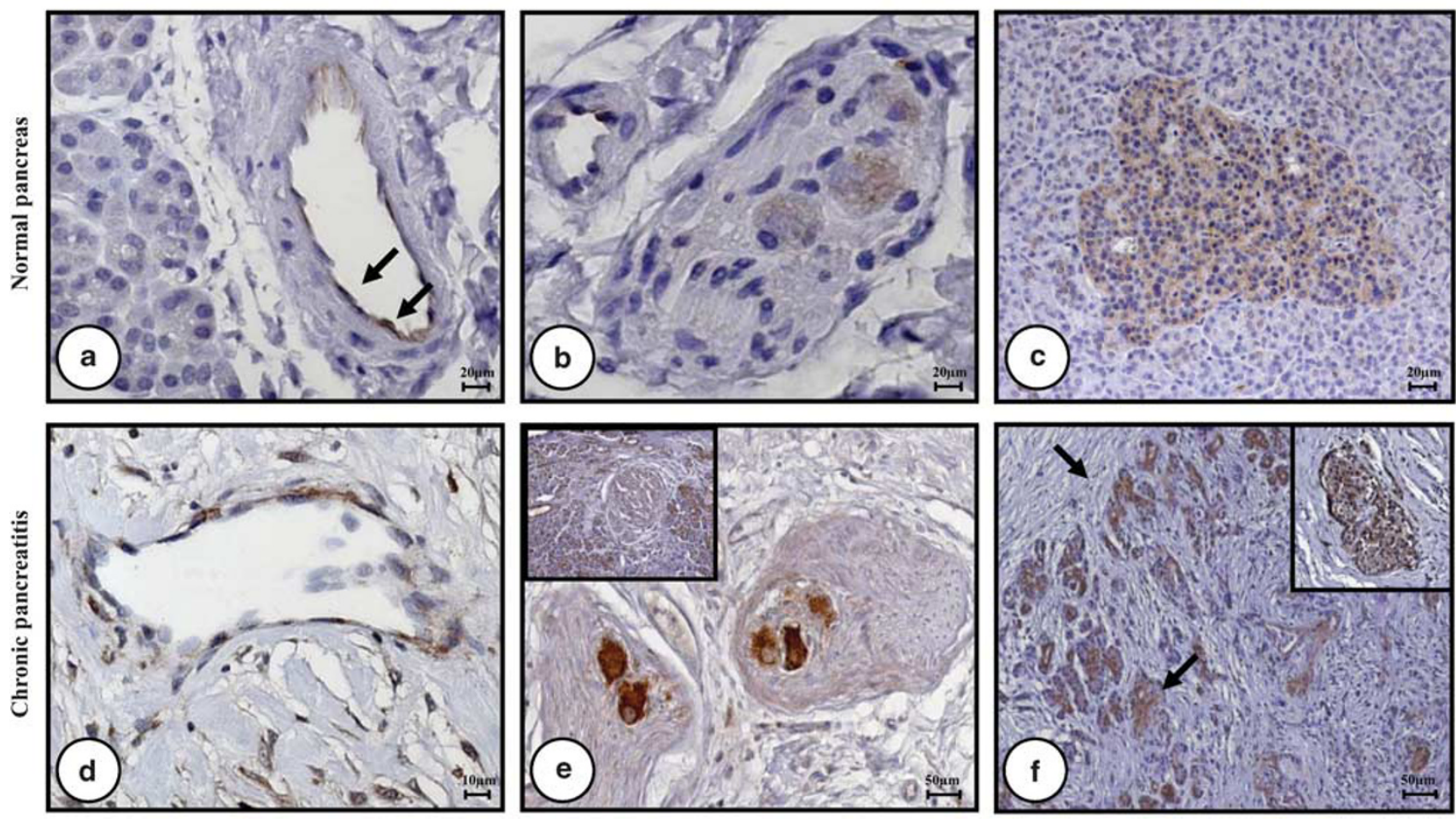

\section{CX3CR1}
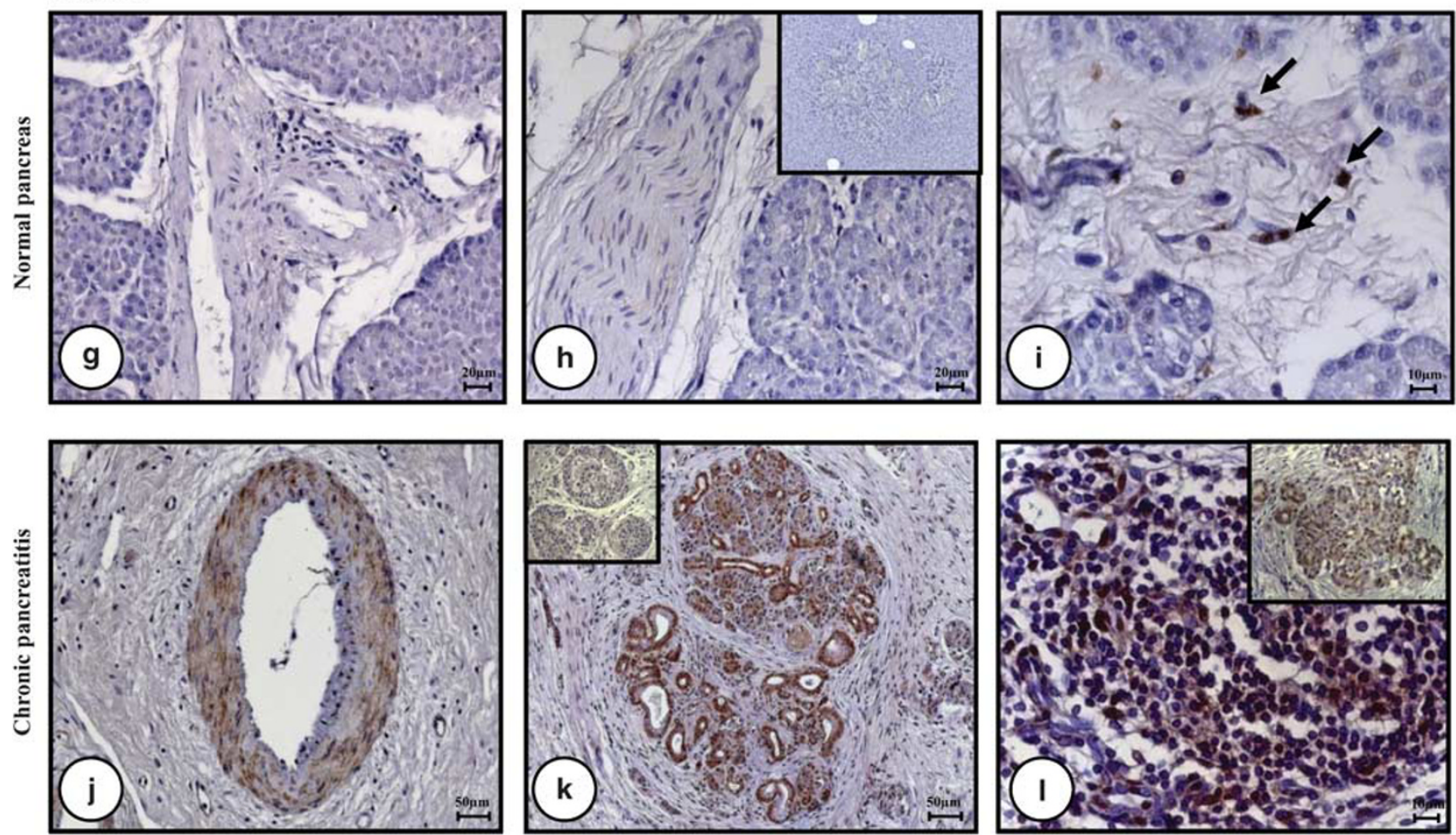

Figure 2 Immunolocalization of fractalkine and CX3CR1: In normal pancreas, fractalkine was weakly present in endothelial cells (see arrows in a) and ganglia, but was strong in islets (a-c). Fractalkine immunoreactivity in chronic pancreatitis (CP) showed moderate-to-strong accumulation in endothelial cells (d), in intrapancreatic ganglia and tubular complexes (d-f). Insets in panel (e) depict immunoreactivity of fractalkine in CP acini and islets (f). CX3CR1 was only weak-to-moderate immunoreactive in inflammatory cells in the normal pancreas (g-i, inset in panel h shows islets in NP). In CP, CX3CR1 immunoreactivity changed noticeably with strong immunoreactivities in arteries, tubular complexes and inflammatory cells (j-I, inset in panel k depicts islets and in panel I acini in $(P)$. 
Table 3 Localization of fractalkine and CX3CR1 in NP and CP

\begin{tabular}{|c|c|c|c|c|c|c|c|}
\hline & Acini & Ductal cells & Tubular complexes & Ganglia & Inflammatory cells & Arteries & Islets \\
\hline \multicolumn{8}{|l|}{$N P$} \\
\hline Fractalkine & - & - & & $-/+$ & - & $-/+$ & ++ \\
\hline$C \times 3 C R 1$ & - & - & & - & $+/++$ & - & - \\
\hline \multicolumn{8}{|l|}{$C P$} \\
\hline Fractalkine & $(+/++)$ & ++ & ++ & +++ & + & ++ & +++ \\
\hline$C \times 3 C R 1$ & $(+/++)$ & +++ & +++ & $++/+++$ & $++/+++$ & $++/+++$ & - \\
\hline
\end{tabular}

$\mathrm{CP}$, chronic pancreatitis; NP, normal pancreas.

Semi-quantitative analysis of fractalkine and $C X 3 C R 1$ immunoreactivity in NP and CP.

- , absent; + , weak; ++ , moderate; +++ , strong immunoreactivity.

immunoreactivity only in the damaged acini (Figure $2 \mathrm{f}$ and $\mathrm{k}$, inset in panel 1; Table 3). The unaffected pancreatic acini showed neither fractalkine nor CX3CR1 immunoreactivity.

\section{Neural Immunoreactivity of Fractalkine and CX3CR1}

In normal pancreas, axons, perineurium and endoneurium were fractalkine-negative with only weak immunoreactivity in intrapancreatic ganglia, whereas CX3CR1 did not show any neural immunoreactivity at all (Figure $2 \mathrm{~b}$ and $\mathrm{h}$ and Table 3). The staining pattern changed in CP with strong fractalkine and moderate-to-strong CX3CR1 immunoreactivity in the intrapancreatic ganglia (Figures $2 \mathrm{e}$ and $3 \mathrm{~h}$ ). As shown in Figure $3 \mathrm{a}-\mathrm{h}$, the immunoreactivity of nerve fibers showed an assortment of diverse intensities for fractalkine and CX3CR1 in different $\mathrm{CP}$ patients. It was remarkable that in nerves without neural infiltration of inflammatory cells, no or weak immunoreactivity of fractalkine and CX3CR1 was detected (Figure 3a, c, e and g), whereas in nerves with perineural or endoneural mononuclear cell infiltration, the neural immunoreactivity for both fractalkine and CX3CR1 was noticeably higher (Figure $3 \mathrm{~b}, \mathrm{~d}, \mathrm{f}$ and $\mathrm{h}$ ).

\section{Fractalkine/CX3CR1 and Pancreatic Neuritis}

The severity of pancreatic neuritis was significantly associated with higher pain scores of the CP patients (Figure 4a). Patients with endoneural inflammatory cell infiltration revealed higher pain scores $(\mathrm{II} / 5.4 \pm 0.5)$ than those without any pancreatic neuritis $(0 / 2.8 \pm 0.8$; Figure $4 a)$. To investigate the possible influence of fractalkine on pancreatic neuritis, we related the severity of pancreatic neuritis to the neural immunoreactivity of fractalkine. Fractalkine neuroimmunoreactivity scores (II/3.9 \pm 0.5$)$ were significantly $(P<0.05)$ stronger in patients with endoneural pancreatic neuritis than those with no pancreatic neuritis $(0 / 1.2 \pm 0.6$; Figure $4 \mathrm{~b}$ ). Correspondingly, increased tissue fractalkine mRNA expression was linked to severe pancreatic neuritis (341 \pm 54 transcripts/ $\mu$ l compared with no neuritis $(141 \pm 27$ transcripts/ $\mu \mathrm{l}$; Figure $4 \mathrm{~d})$. Both neural immunoreactivity and mRNA expression of CX3CR1 tended to increase with the severity of pancreatic neuritis, but did not reach statistical significance (Figure $4 \mathrm{c}$ and e).

\section{Fractalkine/CX3CR1 and Pancreatic Pain}

In the investigated collective, 15 patients had no pain (Pain 0), 8 patients had mild pain (Pain I) and 38 patients had moderate-to-severe pain (Pain II). Fractalkine mRNA expression was positively related to the severity of pain in CP and was significantly $(P<0.05)$ increased in patients with severe pain (Pain II, $325 \pm 42$ transcripts/ $\mu$ l) compared with those without pain (Pain $0,147 \pm 21$ transcripts/ $\mu$; Figure 5a). CX3CR1 was also increased in the Pain II group compared with the Pain 0 group, but failed to reach statistical significance (Figure $5 b$ ). These results were confirmed on the protein level: the fractalkine protein level was positively related to the severity of pain in $\mathrm{CP}$ and was increased in patients with severe pain (Pain II, 4.7-fold) compared with those without pain (Pain 0, Figure 5c). Similarly, the CX3CR1 protein content was also increased in the Pain II group compared with the Pain 0 group (3.5-fold, Figure 5d). Furthermore, the duration of CP-associated complaints was significantly related to the neural immunoreactivity of fractalkine (Table 4). Patients with the longest history of $\mathrm{CP}$ showed the strongest fractalkine neuro-immunoreactivity. This phenomenon was not observed with CX3CR1 (Table 4).

To study, whether neural fractalkine immunoreactivity was interfering with the pain sensation, fractalkine and CX3CR1 were related to the severity of pain. Fractalkine and CX3CR1 neural immunoreactivity revealed a significant association with the pain sensation in $\mathrm{CP}$ (Figure $5 \mathrm{e}$ and $\mathrm{f}$ ). Fractalkine $(P<0.002)$ and $C X 3 C R 1 \quad(P<0.001)$ neural immunoreactivity was significantly increased in patients with severe pain (Pain II) when compared with those without pain (Pain 0) (Figure 5e and f). CX3CR1 immunoreactivity even differed significantly $(P<0.05)$ between the moderate Pain I and severe Pain II group (Figure 5f). 

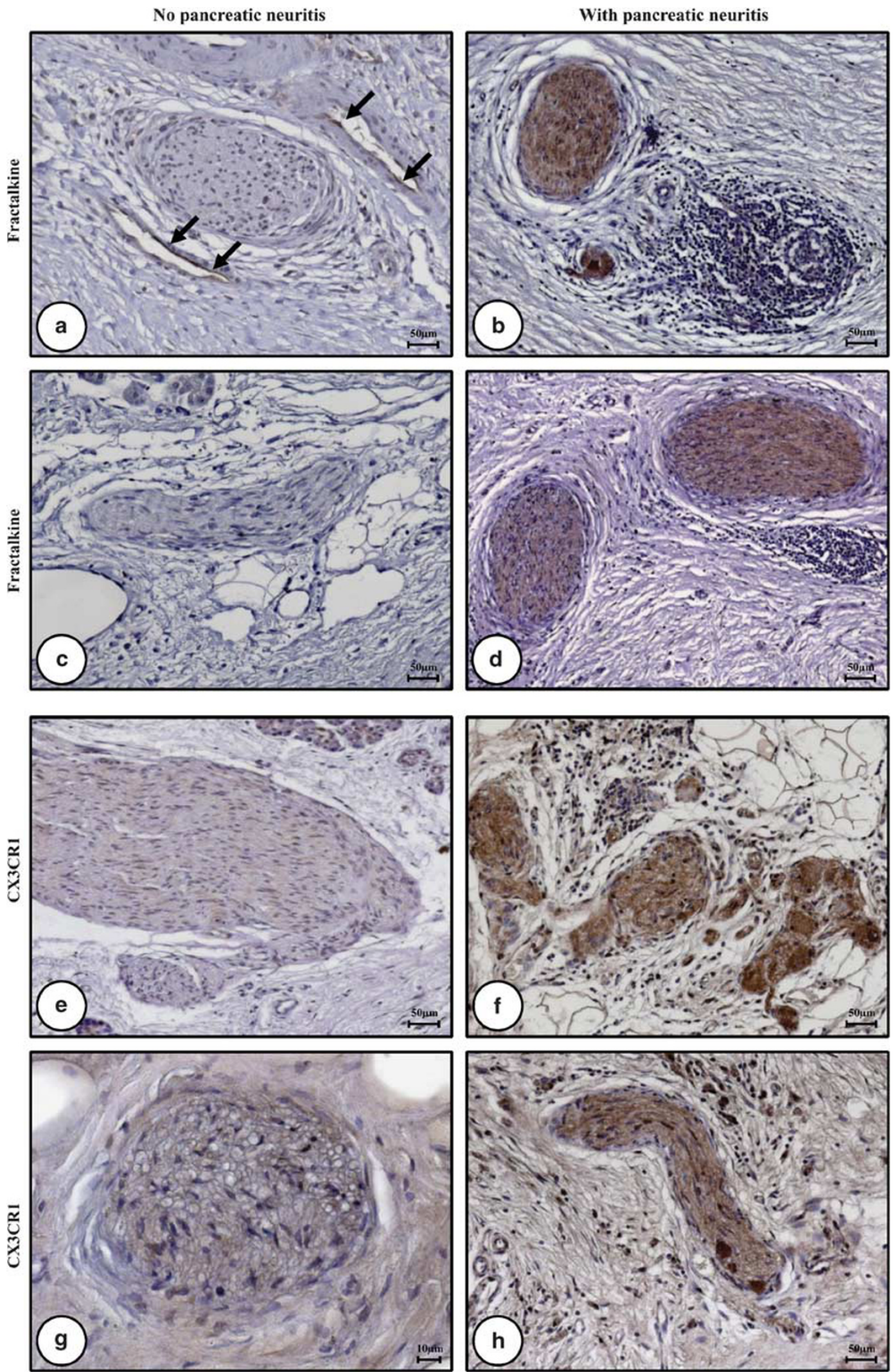

Figure $3 \mathrm{In} \mathrm{CP}$, immunoreactivity of fractalkine (b and $\mathbf{d}$ ) and CX3CR1 (f and $\mathbf{h}$ ) was markedly increased in intrapancreatic nerves with inflammatory cell infiltration (pancreatic neuritis) than without ( $\mathbf{a}, \mathbf{c}$ and $\mathbf{e}, \mathbf{g}$, respectively). (a) Endothelial cells immunoreactive (see arrows) for fractalkine, but missing neural immunoreactivity. 

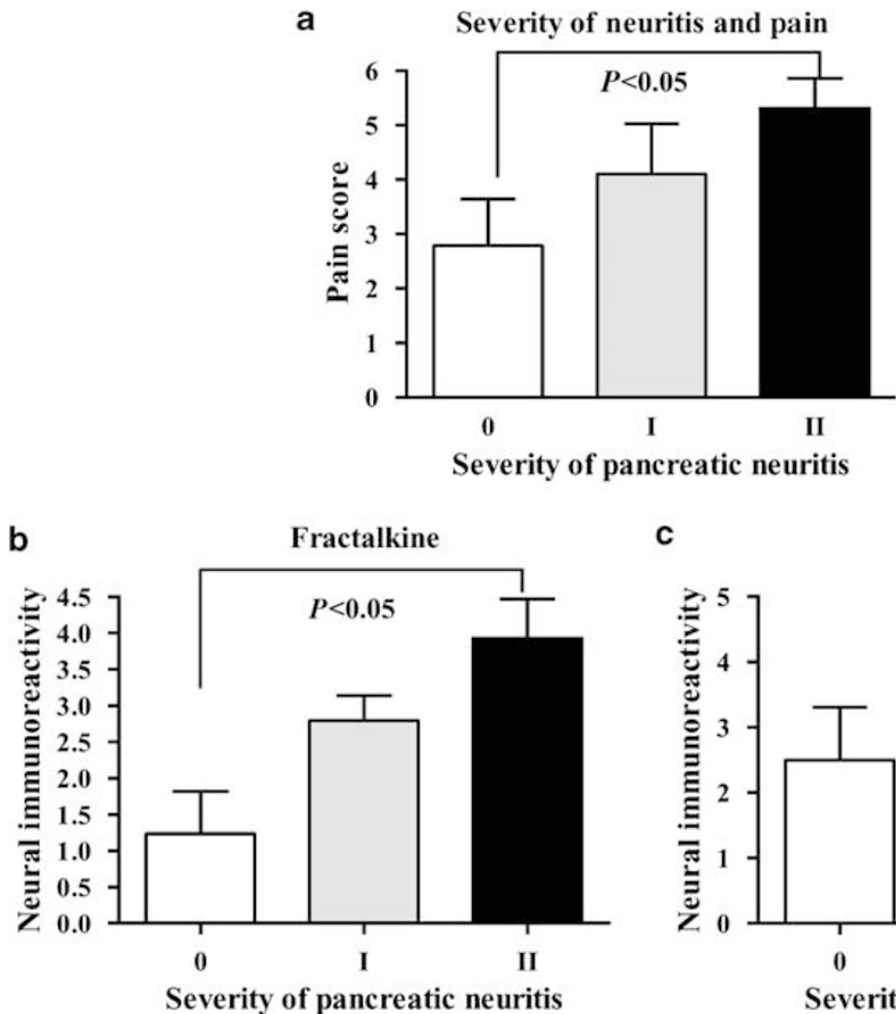

c

CX3CR1

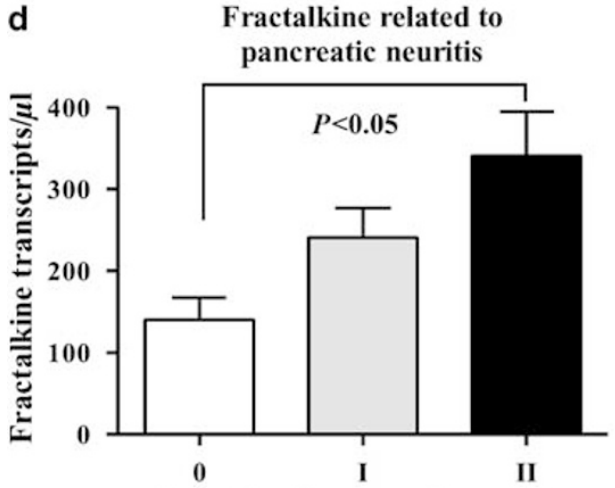

Severity of pancreatic neuritis

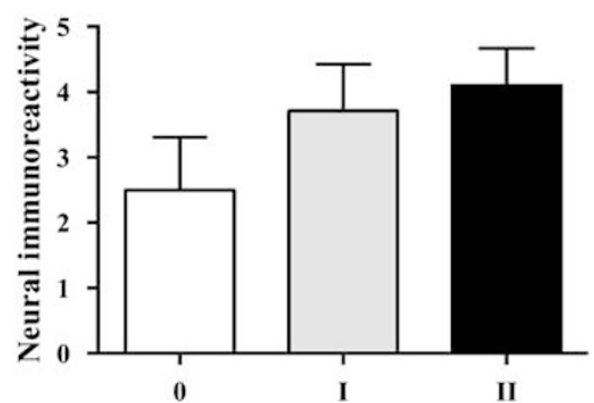

Severity of pancreatic neuritis

e

CX3CR1 related to pancreatic neuritis

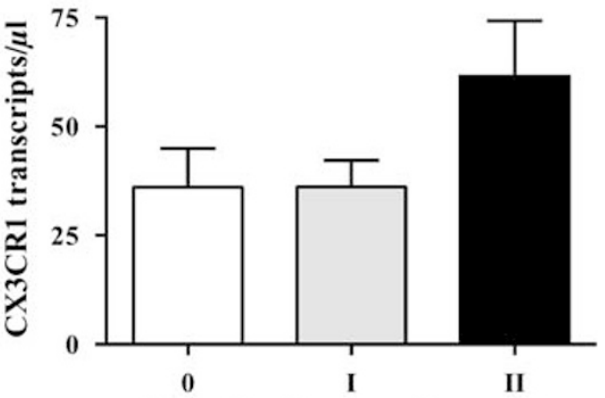

Severity of pancreatic neuritis

Figure 4 (a) The severity of pancreatic neuritis was significantly associated with pain sensation in CP. (b) Neuro-immunoreactivity of fractalkine was closely linked to the severity of pancreatic neuritis, whereas (c) CX3CR1 neuro-immunoreactivity tended to increase with enhanced pancreatic neuritis, but failed to reach statistical significance. Correspondingly, tissue (d) fractalkine mRNA expression was also associated with the severity of pancreatic neuritis, and again (e) $C X 3 C R 1$ transcripts showed the same trend, but was again not significant.

\section{Fractalkine/CX3CR1 and Neural Alterations in CP}

Owing to the prominent neural activity of fractalkine and CX3CR1 in CP, we studied their possible influence on CPassociated neuropathic changes, characterized by increased nerve fiber density and hypertrophy. A quantitative analysis revealed an evident increase in the nerve fiber density and total nerve area in $\mathrm{CP}$ compared with NP, as reported before $(P<0.001$, data not shown $){ }^{31,33}$ However, fractalkine and CX3CR1 mRNA expressions and neural immunoreactivity did not correlate with changes in nerve fiber density and hypertrophy in CP (data not shown).

\section{Fractalkine/CX3CR1 and Inflammatory Cell Infiltration in} CP

To investigate the possible influences of fractalkine and CX3CR1 expressions on lymphocyte and macrophage infiltration into the damaged CP tissue, mononuclear cells were labeled with $C D 45$ and $C D 68$, respectively. The area of infiltrating CD45-positive lymphocytes in CP correlated positively with both fractalkine $(P<0.001$, Figure $6 a)$ and CX3CR1 mRNA expressions $(P<0.02$, Figure $6 \mathrm{~b})$. Fractalkine mRNA levels did not correlate with the area of CD68-positive cells $(P=0.1$, Figure $6 \mathrm{c})$, whereas $C X 3 C R 1$ 

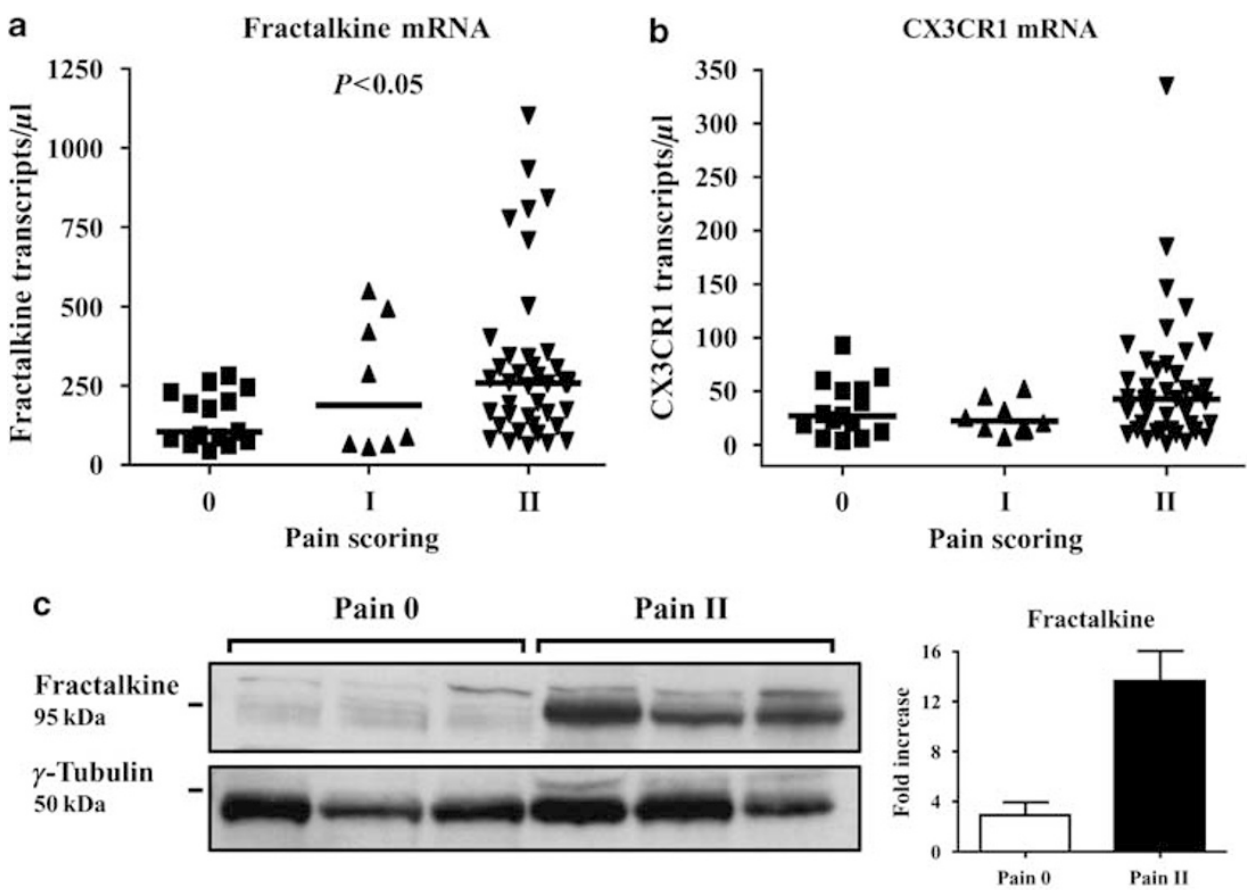

d

Pain 0

Pain II
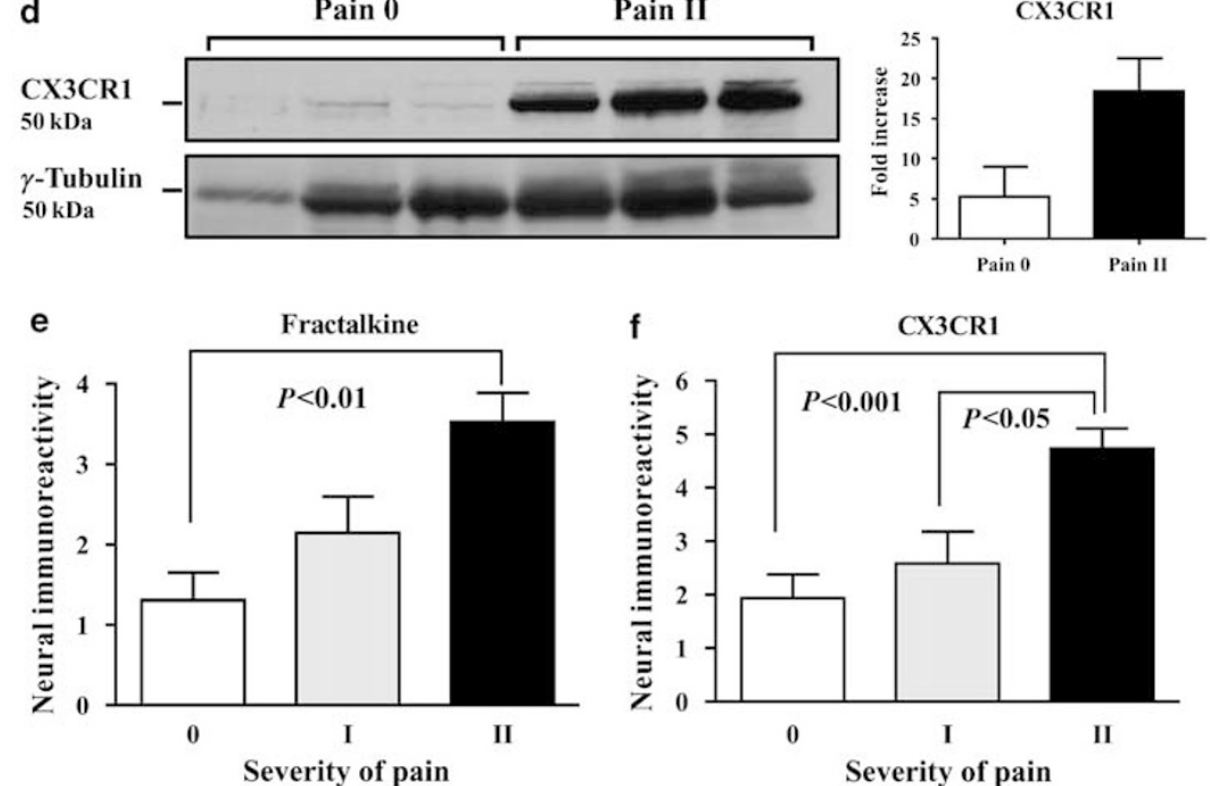

Figure 5 (a) Fractalkine mRNA expression was positively related to the severity of pain in $C P(P<0.05)$, whereas $C X 3 C R 1$ tended to increase in $C P$, but failed to reach statistical significance $(\mathbf{b})$. Western blot analysis identified an increase in fractalkine (c) and $C X 3 C R 1$ (d) in patients with moderate-to-severe pain (Pain II; $n=3$ ) compared with those without pain (Pain $0 ; n=3$ ). Equal protein loading is verified with $\gamma$-tubulin. Neural immunoreactivity of fractalkine (e) and $C X 3 C R 1$ (f) was significantly related to the severity of pain in patients with CP. Data are presented as mean \pm s.e.m.

mRNA expression $(P<0.0001$, Figure $6 \mathrm{~d})$ presented a highly significant correlation with the infiltration area of macrophages in CP. To evaluate whether the intrapancreatic infiltration of lymphocytes and especially macrophages are involved in pain sensation, the immunoreactivity of CD45 and $C D 68$ was related to pain severity in $\mathrm{CP}$ patients. The percentage of CD45-positive lymphocytes was positively related to the severity of pain in $\mathrm{CP}$, whereas $C D 68$-positive macrophages did not show any significant association with pain sensation (Figure 6e and $\mathrm{f}$ ).

\section{Fractalkine and Fibrosis in $\mathrm{CP}$}

The degree of fibrotic tissue remodeling in $\mathrm{CP}$ was closely associated with pain sensation in CP patients (Figure 7a). Patients with severe and frequent pain $(9.2 \pm 0.5)$ showed the worse fibrotic tissue reactions when compared with $\mathrm{CP}$ 
Table 4 Correlation analysis of fractalkine/CX3CR1 neuro-immunoreactivity with disease duration of CP

\begin{tabular}{lcl}
\hline & \multicolumn{2}{c}{ CP disease duration } \\
\cline { 2 - 3 } & $r$-value & $P$-value \\
\hline Fractalkine & 0.65 & 0.002 \\
CX3CR1 & 0.15 & 0.45
\end{tabular}

$\mathrm{CP}$, chronic pancreatitis.

$r$-value: Spearman's correlation coefficient.

patients with no pain sensations at all $(4.6 \pm 1.2$; Figure $7 \mathrm{a})$. Fractalkine mRNA expression was associated with the severity of fibrosis in CP, whereas CX3CR1 failed to reach statistical significance (Figure $7 \mathrm{~b}$ and c). Fractalkine expression showed a significant $(P<0.01)$ increase in the fibrosis group II (346 \pm 51 transcripts $/ \mu \mathrm{l})$ compared with group 0 (114 \pm 75 transcripts/ $\mu$ l, Figure $7 \mathrm{~b})$. To evaluate the putative impact of fractalkine on fibrogenesis in $\mathrm{CP}$, isolated hPSCs were incubated with different concentrations of fractalkine. Stimulation with fractalkine for $24 \mathrm{~h}$ decreased the expression of $\alpha$-SMA and Collagen-1 mRNA in isolated hPSCs in a nonsignificant manner (data not shown). In the hPSC lysates, the $\alpha$-SMA and Collagen- 1 content did not change (Figure 7d). Corresponding to the mRNA data, Collagen-1 in the hPSC supernatants gradually decreased with higher concentrations of fractalkine (Figure 7e).

\section{DISCUSSION}

The currently accepted definition for neuropathic pain is 'pain arising as a direct consequence of a lesion or disease affecting the somato-sensory system. ${ }^{34}$ Although pain in CP would traditionally be classified as inflammatory pain, recent studies pointed to the simultaneous presence of structural changes in axons and ganglia in $\mathrm{CP}$, thus implying the involvement of a concomitant neuropathic component. ${ }^{35,36,58}$ Previous studies have determined that the 'inflammatory' and the 'neuropathic' component of pain in $\mathrm{CP}$ may not be independent but instead closely linked: The 'neuro-immune crosstalk' through pancreatic neuritis, and changes in intrapancreatic neural plasticity were all associated with the severity of pain experienced by CP patients. ${ }^{8,10,33,58}$ In addition, recent studies support the idea that the peripheral immune system and spinal microglia are crucial in the generation of neuropathic pain. ${ }^{35,37,58}$

The contribution of fractalkine to general neuropathic pain was shown earlier: In several experimental models of neuropathic pain, it was shown that endogenous fractalkine is released by DRG and is accompanied by the upregulation of CX3CR1 in the spinal microglia and neuropathic pain behavior. ${ }^{12,14,23,24,38,39}$ Similar effects were observed when fractalkine was administered exogenously. ${ }^{40}$ In both cases, neutralizing antibodies to $C X 3 C R 1$ blocked and attenuated neuropathic pain. ${ }^{23,24,39-42}$ A recent spared nerve injury model of neuropathic pain in mice showed attenuation of neuropathic pain-related behavior after intraneural injection of fractalkine into the sciatic nerve. ${ }^{27}$ Although a discrepancy between the central pro-algesic and peripheral anti-algesic effect of fractalkine is a possibility, these studies once again underline the pain-modulatory feature of fractalkine.

Fractalkine is released by neurons after a nerve injury, attracts neutrophilic granulocytes to the sites of damage and leads to macrophage infiltration..$^{23,37,43}$ One can imagine that fractalkine might be inducing neural inflammatory cell infiltration in $\mathrm{CP}$ after its release from intrapancreatic nerves, and thus be a key mediator of pancreatic neuritis. The participation of a chemokine as the mediator in this process is supported by the fact that increasing inflammatory cell infiltration around sympathetic nerve fibers is in part triggered by cytokine and chemokine release by macrophages. ${ }^{44}$ On the basis of our results, in CP, fractalkine seems to be involved in the recruitment of mononuclear cells to the pancreas. However, without the presence of a mediator between the intraneural and extraneural milieu, the sole presence of mononuclear cells in the pancreatic tissue may not be sufficient to both sensitize nociceptors and to induce neuropathic pain. Although fractalkine originating from intrapancreatic nerves might well be such a mediator, it would not be able to reach the extraneural milieu unless the barrier function of the perineurium is lost. Here, one should note that damage to the perineurium of intrapancreatic nerves is a frequently observed phenomenon in $\mathrm{CP}^{8}$ One can imagine that once this barrier function is lost, neural fractalkine would exit the intraneural space and enhance mononuclear cell infiltration by acting as a chemoattractant ('inside-out effect'). On the other hand, inflammatory mediators released by mononuclear cells, such as TNF- $\alpha$ or $I L-1$, can gain access to the axons through the damaged perineural barrier ('outside-in effect') and induce neuropathic pain. ${ }^{45,46}$ Considering the presented data, it does not seem to be surprising that the severity of pancreatic neuritis and the severity of pain are closely related to neuronal fractalkine expression. Our results suggest that neuro-immune interactions are also of major importance for the development and maintenance of visceral pain in CP as proposed earlier for neuropathic pain after somatic nerve damage. ${ }^{47}$ The lack of a correlation between CD68 and pain sensation does not necessarily exclude a role for macrophages in pain in CP. In fact, the levels of pituitary adenylate cyclase-activating polypeptide (PACAP) secreted by macrophages was earlier shown to correlate with pain sensed by CP patients. ${ }^{48}$ Similarly, there was a strong immunoreactivity for PACAP in the perineurium of hypertrophic nerves and in the areas of high immune cell infiltration. In this regard, future studies should aim at determining the distribution of immune cell subtypes infiltrating the nerves, that are neuritis-associated immune cells. 
a

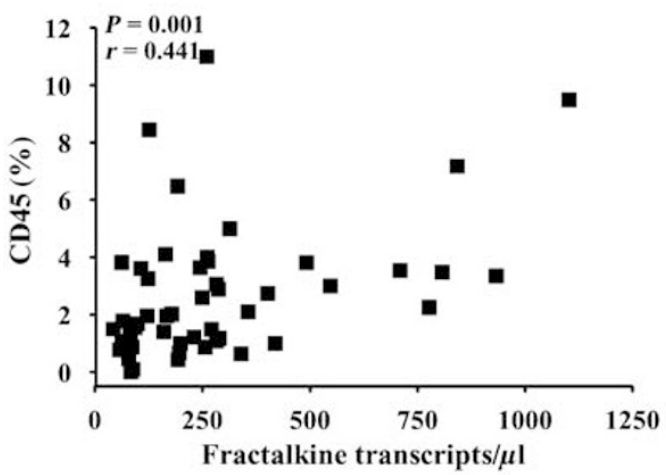

C

Fractalkine correlated with CD68

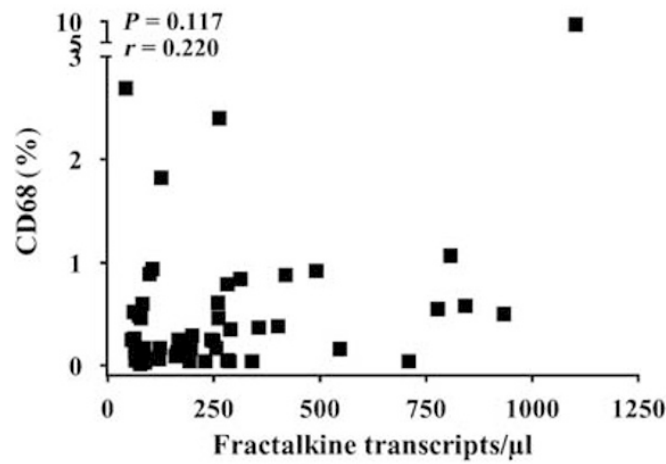

e

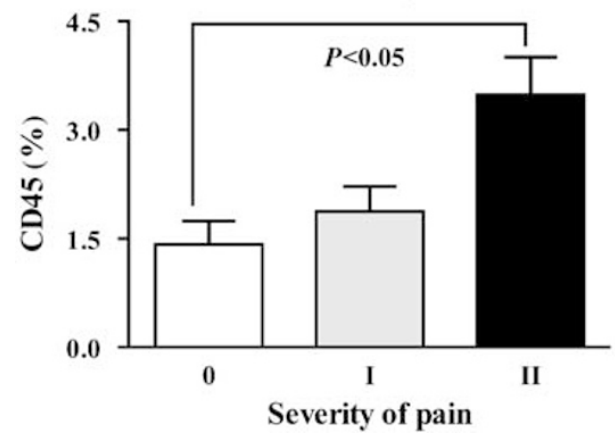

b

CX3CR1 correlated with CD45

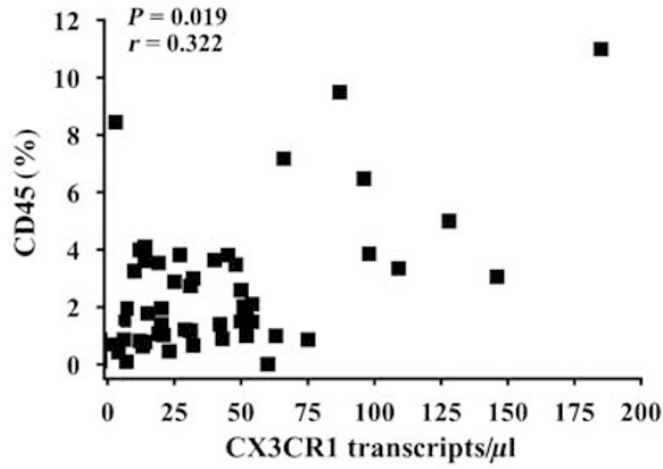

d

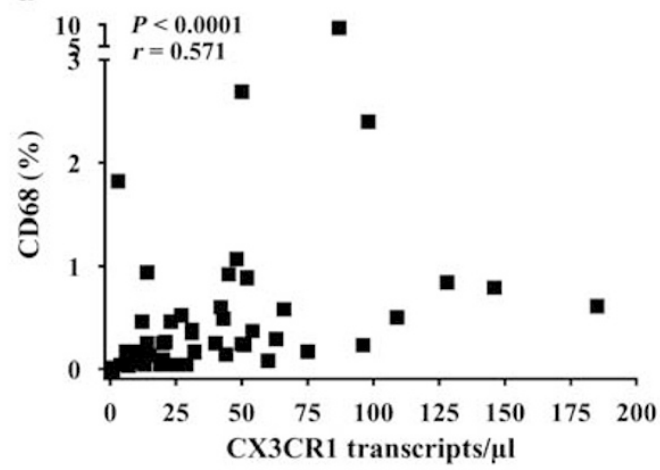

$f \quad \mathrm{CD68}$ related to pain in CP

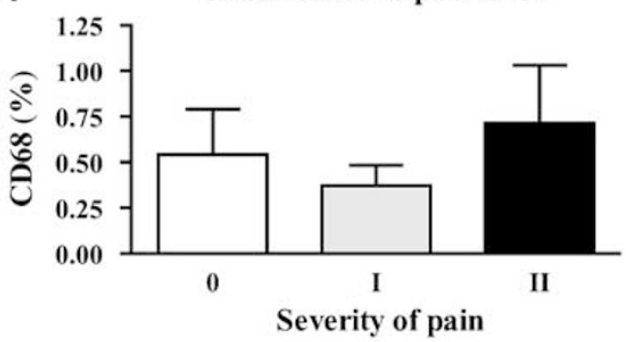

Figure 6 Fractalkine and CX3CR1 mRNA expressions correlated with inflammatory cells immunopositive for CD45 and CD68. The number of infiltrated CD45positive cells in CP correlated positively with both fractalkine (a) and CX3CR1 (b). The number of infiltrating macrophages correlated significantly higher with CX3CR1 mRNA expression (d), but not with fractalkine (c). (e) CD45-positive inflammatory cell infiltration was positively related to the severity of pain in CP, but (f) CD68-positive macrophages did not show any relation to pain. Data are presented as mean \pm s.e.m.

There is increasing body of evidence that pain in $\mathrm{CP}$ is of neuropathic origin. ${ }^{58}$ Although several components of pancreatic neuropathy, including increased neural density and hypertrophy, were shown to be linked to pain sensation in $\mathrm{CP}$, the role of another possible contributor, namely glia cells in $\mathrm{CP}$, remains uninvestigated. In $\mathrm{CP}$, increased neural and tissue fractalkine did not account for all the mechanisms of pain in $\mathrm{CP}$, as changes in intrapancreatic neural plasticity were found as fractalkine-unrelated correlates of pain. The key role of glia cells, including Schwann cells in the peripheral nervous system, is well established in several neuropathic pain states. ${ }^{37}$ In this regard, the very recently recognized role of fractalkine in neuropathic pain through microglial activation deserves special consideration. ${ }^{49}$ Indeed, although our study lacks a correlation between fractalkine expression and the morphological correlates of neural density and hypertrophy, fractalkine may well be exerting its neuropathic painmodulatory effect through the activation of intrapancreatic Schwann cells, a possibility that certainly deserves to be a subject of future studies. With this study, we show, for the first time, fractalkine as an additional key player in clinical visceral pain. Moreover, the higher average fractalkine neural immunoreactivity in patients with a longer standing history of $\mathrm{CP}$ brings up the idea that there might be an accumulation 

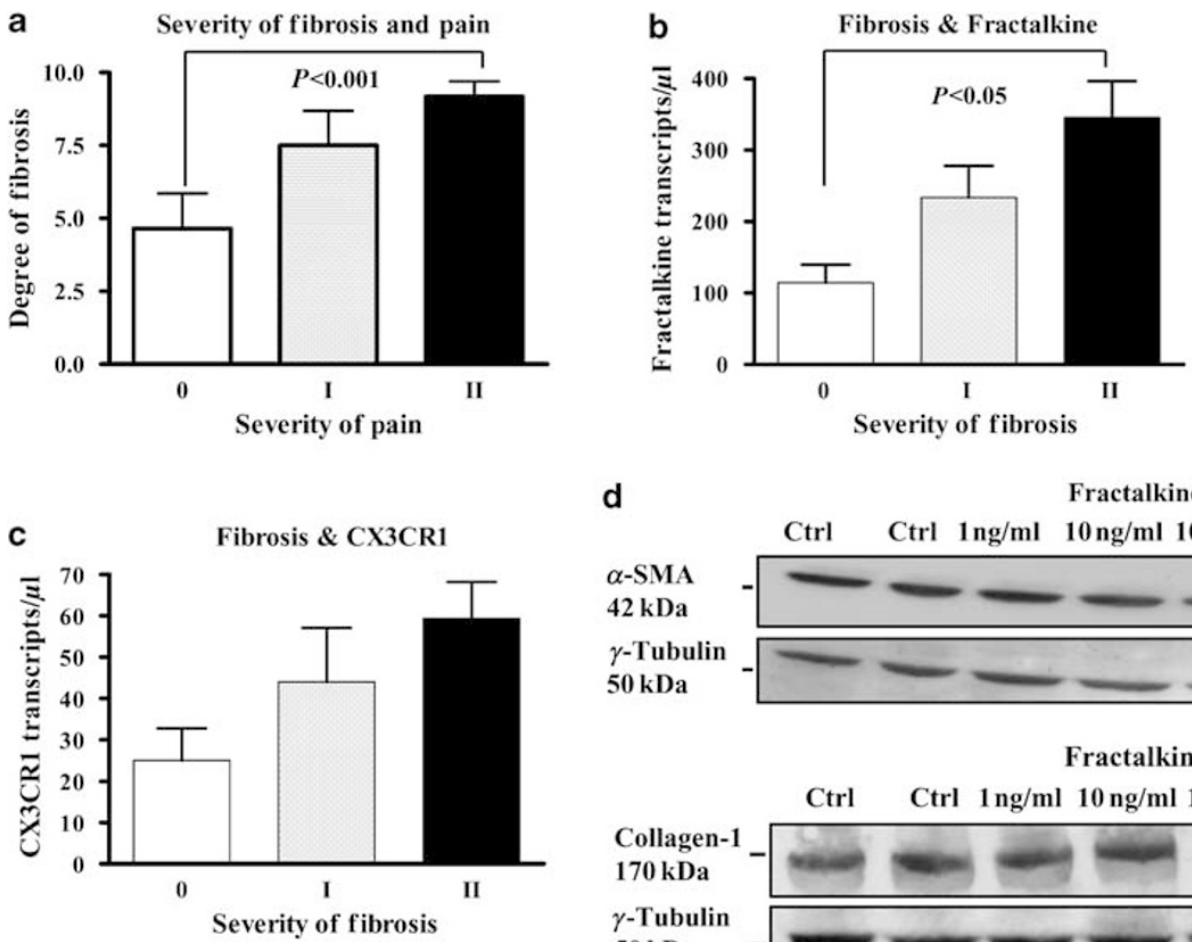

d

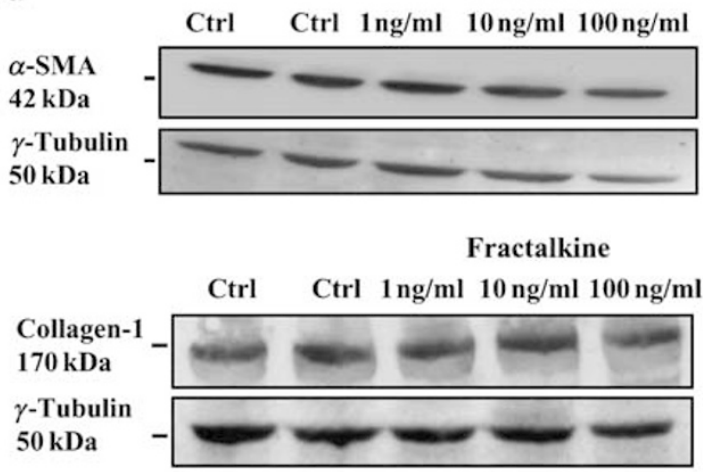

e

Fractalkine

Ctrl Ctrl $1 \mathrm{ng} / \mathrm{ml} 10 \mathrm{ng} / \mathrm{ml} 100 \mathrm{ng} / \mathrm{ml}$

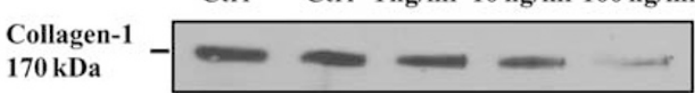

Figure 7 (a) The severity of fibrosis was significantly linked to the pain sensation of CP patients. (b) Fractalkine mRNA expression was associated with the severity of fibrosis in chronic pancreatitis, whereas (c) CX3CR1 failed to reach statistical significance. Isolated human pancreatic stellate cells (hPSC) were incubated with different concentrations of fractalkine at different time points, followed by $\alpha$-SMA and Collagen-1 mRNA, and protein detection. (d) In the hPSCs lysates, no changes on protein level were detected for $\alpha$-SMA and Collagen- 1 after fractalkine stimulation. Equal protein loading was verified with $\gamma$ tubulin. (e) Corresponding to the mRNA data, Collagen-1 in the hPSC supernatants gradually decreased with higher concentrations of fractalkine.

of fractalkine in intrapancreatic nerves of $\mathrm{CP}$ patients over time. This contribution of fractalkine seems to distinguish it from our control chemokine, the $M C P-1$, which is secreted by macrophages at sites of neural injury. ${ }^{50}$ Contrary to fractalkine, $M C P-1$ expression did not show any significant association with pain sensation in our study (data not shown). However, whether this upregulation of fractalkine and CX3CR1 in CP is a pro-algesic or a counter-regulatory anti-algesic mechanism remains unknown, and should be a subject for future investigations.

Cytokines, such as $T N F-\alpha$, and chemokines, such as $I L-1$, IL-6 and MCP-1, are not only related to nociceptor sensitization but also involved in the development of fibrosis in $\mathrm{CP}$ by interfering in hPSCs proliferation and ECM metabolism. ${ }^{51-53}$ As a result of increased recruitment of inflammatory cells, activated mononuclear cells release profibrotic cytokines and chemokines, such as TGF- $\beta$, TNF- $\alpha$ and $M C P-1$, which in turn activate hPSCs and promote fibrosis in $C P{ }^{4,52,53}$ The chemokine $M C P-1$, one of the major monocyte chemoattractants, is highly expressed in fibroblasts in CP, induces TGF- $\beta$ upregulation and is associated with the progression of pancreatic fibrosis. ${ }^{52,54}$ Blocking of $M C P-1$ attenuates the degree of fibrosis in experimental $\mathrm{CP}^{52}$ Regarding fractalkine and fibrosis, CX3CR1 was identified to have a genetic and functional importance in higher stages of liver fibrosis in chronic hepatitis $\mathrm{C}$, and was noticeably upregulated in patients with enhanced renal fibrosis. ${ }^{21,22}$ In experimental post-ischemic renal fibrosis, fractalkine and CX3CR1 expression levels were associated with enhanced inflammation and fibrosis, which could be diminished by blocking antibodies to CX3CR1 through the reduction of $\alpha-S M A$ and Collagen-1. ${ }^{55}$ In human $\mathrm{CP}$, this interaction appears to be different. Although fractalkine was associated with the severity of fibrosis, fractalkine had no significant impact on the activation of hPSCs, and even tended to reduce the activation of isolated hPSCs by decreasing the mRNA expression of $\alpha$-SMA and especially that of Collagen-1. Regarding our presented data, fractalkine does not seem to be directly involved in the fibrogenesis processes in $\mathrm{CP}$, as it was observed in renal and liver fibrosis. ${ }^{21,22}$ In all likelihood, it 
promotes tissue fibrosis indirectly by increasing the mononuclear cell infiltration and not through the activation of hPSCs. This hypothesis is supported by recent reports: (1) Ishida and colleagues $^{56}$ showed that CX3CR1 depletion induced a marked reduction in the number of macrophages and in the release of TGF- $\beta 1$, and observed reduced $\alpha-S M A$ and Collagen-1 depositions in the skin from wounded $C X 3 C R 1^{-1-}$ mice. (II) Considering that macrophages activate stellate cells and that suppression of macrophage infiltration inhibits hepatic stellate cell activation and liver fibrosis, the fractalkine/CX3CR1-triggered macrophage tissue infiltration might represent another possible contributor to fibrogenesis in $\mathrm{CP}^{57}$

In conclusion, as a result of intrapancreatic neuropathic changes in $\mathrm{CP}$, fractalkine may be involved in pain modulation through neural activation and through the attraction of inflammatory cells to intrapancreatic nerves. Fractalkine is exceptional among other chemokines because it binds selectively to the CX3CR1, and this receptor binds only to fractalkine. ${ }^{13,15,33}$ Thus, it is an interesting idea that blocking a single chemokine receptor might influence abdominal pain sensation, inflammation and may be also fibrosis in CP. Therefore, further studies are needed to elucidate the particular role of CX3CR1 in CP and to assess its potential role as a novel therapeutic target.

\section{ACKNOWLEDGEMENT}

The authors are grateful to Ms Kathrin Schneider, Ms Monika Meinhardt, Ms Brunhilde Bentzinger and Mr Burak Altintas for their superior technical support.

1. Dimagno EP. A short, eclectic history of exocrine pancreatic insufficiency and chronic pancreatitis. Gastroenterology 1993;104:1255-1262.

2. Sarles H, Bernard JP, Johnson C. Pathogenesis and epidemiology of chronic pancreatitis. Annu Rev Med 1989;40:453-468.

3. Adler G, Schmid RM. Chronic pancreatitis: still puzzling? Gastroenterology 1997;112:1762-1765.

4. Michalski CW, Gorbachevski A, Erkan M, et al. Mononuclear cells modulate the activity of pancreatic stellate cells which in turn promote fibrosis and inflammation in chronic pancreatitis. J Transl Med 2007;5:63.

5. Bachem MG, Schneider $\mathrm{E}$, Gross $\mathrm{H}$, et al. Identification, culture, and characterization of pancreatic stellate cells in rats and humans. Gastroenterology 1998;115:421-432.

6. Apte MV, Wilson JS. Mechanisms of pancreatic fibrosis. Dig Dis 2004;22:273-279.

7. Kloppel G, Detlefsen S, Feyerabend B. Fibrosis of the pancreas: the initial tissue damage and the resulting pattern. Virchows Arch 2004:445:1-8.

8. Bockman DE, Buchler M, Malfertheiner $P$, et al. Analysis of nerves in chronic pancreatitis. Gastroenterology 1988;94:1459-1469.

9. Keith RG, Keshavjee SH, Kerenyi NR. Neuropathology of chronic pancreatitis in humans. Can J Surg 1985;28:207-211.

10. Di Sebastiano $P$, Fink $T$, Weihe $E$, et al. Immune cell infiltration and growth-associated protein 43 expression correlate with pain in chronic pancreatitis. Gastroenterology 1997;112:1648-1655.

11. Bazan JF, Bacon KB, Hardiman G, et al. A new class of membranebound chemokine with a CX3C motif. Nature 1997;385:640-644.

12. Lindia JA, McGowan $\mathrm{E}$, Jochnowitz $\mathrm{N}$, et al. Induction of CX3CL1 expression in astrocytes and CX3CR1 in microglia in the spinal cord of a rat model of neuropathic pain. J Pain 2005;6:434-438.
13. Asensio VC, Campbell IL. Chemokines in the CNS: plurifunctional mediators in diverse states. Trends Neurosci 1999;22:504-512.

14. Verge GM, Milligan ED, Maier SF, et al. Fractalkine (CX3CL1) and fractalkine receptor (CX3CR1) distribution in spinal cord and dorsal root ganglia under basal and neuropathic pain conditions. Eur J Neurosci 2004;20:1150-1160.

15. Hughes PM, Botham MS, Frentzel $S$, et al. Expression of fractalkine (CX3CL1) and its receptor, CX3CR1, during acute and chronic inflammation in the rodent CNS. Glia 2002;37:314-327.

16. Umehara $\mathrm{H}$, Bloom ET, Okazaki $\mathrm{T}$, et al. Fractalkine in vascular biology: from basic research to clinical disease. Arterioscler Thromb Vasc Biol 2004;24:34-40.

17. Deiva K, Geeraerts $T$, Salim $H$, et al. Fractalkine reduces $N$-methylD-aspartate-induced calcium flux and apoptosis in human neurons through extracellular signal-regulated kinase activation. Eur J Neurosci 2004;20:3222-3232.

18. Yoneda O, Imai T, Goda S, et al. Fractalkine-mediated endothelial cell injury by NK cells. J Immunol 2000;164:4055-4062.

19. Imai T, Hieshima $K$, Haskell $C$, et al. Identification and molecular characterization of fractalkine receptor CX3CR1, which mediates both leukocyte migration and adhesion. Cell 1997;91:521-530.

20. Goda S, Imai T, Yoshie O, et al. CX3C-chemokine, fractalkine-enhanced adhesion of THP-1 cells to endothelial cells through integrindependent and -independent mechanisms. J Immunol 2000;164: 4313-4320.

21. Koziolek MJ, Schmid H, Cohen CD, et al. Potential role of fractalkine receptor expression in human renal fibrogenesis. Kidney Int 2007;72:599-607.

22. Wasmuth $\mathrm{HE}$, Zaldivar MM, Berres $\mathrm{ML}$, et al. The fractalkine receptor CX3CR1 is involved in liver fibrosis due to chronic hepatitis $\mathrm{C}$ infection. J Hepatol 2007;48:208-215.

23. Zhuang ZY, Kawasaki Y, Tan PH, et al. Role of the CX3CR1/p38 MAPK pathway in spinal microglia for the development of neuropathic pain following nerve injury-induced cleavage of fractalkine. Brain Behav Immun 2006;21:642-651.

24. Sun S, Cao H, Han M, et al. New evidence for the involvement of spinal fractalkine receptor in pain facilitation and spinal glial activation in rat model of monoarthritis. Pain 2007;129:64-75; e-pub 2006 Nov 22.

25. Zelenka M, Schafers M, Sommer C. Intraneural injection of interleukin1 beta and tumor necrosis factor-alpha into rat sciatic nerve at physiological doses induces signs of neuropathic pain. Pain 2005; 116:257-263.

26. Gazda LS, Milligan ED, Hansen MK, et al. Sciatic inflammatory neuritis (SIN): behavioral allodynia is paralleled by peri-sciatic proinflammatory cytokine and superoxide production. J Peripher Nerv Syst 2001;6: 111-129.

27. Holmes FE, Arnott N, Vanderplank P, et al. Intra-neural administration of fractalkine attenuates neuropathic pain-related behaviour. J Neurochem 2008;106:640-649.

28. Erkan M, Kleeff J, Esposito l, et al. Loss of BNIP3 expression is a late event in pancreatic cancer contributing to chemoresistance and worsened prognosis. Oncogene 2005;24:4421-4432.

29. Koninger J, Balaz $P$, Wagner $M$, et al. Phosphatidylserine receptor in chronic pancreatitis: evidence for a macrophage independent role. Ann Surg 2005;241:144-151.

30. Erkan M, Kleeff J, Gorbachevski A, et al. Periostin creates a tumorsupportive microenvironment in the pancreas by sustaining fibrogenic stellate cell activity. Gastroenterology 2007;132:1447-1464.

31. Ceyhan GO, Bergmann F, Kadihasanoglu M, et al. The neurotrophic factor artemin influences the extent of neural damage and growth in chronic pancreatitis. Gut 2007;56:534-544.

32. Ceyhan GO, Giese NA, Erkan M, et al. The neurotrophic factor artemin promotes pancreatic cancer invasion. Ann Surg 2006;244:274-281.

33. Jung S, Aliberti J, Graemmel P, et al. Analysis of fractalkine receptor CX(3)CR1 function by targeted deletion and green fluorescent protein reporter gene insertion. Mol Cell Biol 2000;20:4106-4114.

34. Treede RD, Jensen TS, Campbell JN, et al. Neuropathic pain: redefinition and a grading system for clinical and research purposes. Neurology 2008;70:1630-1635.

35. Ceyhan GO, Michalski CW, Demir IE, et al. Pancreatic pain. Best Pract Res Clin Gastroenterol 2008;22:31-44.

36. Drewes $\mathrm{AM}, \mathrm{Krarup} \mathrm{AL}$, Detlefsen $\mathrm{S}$, et al. Pain in chronic pancreatitis: the role of neuropathic pain mechanisms. Gut 2008;57:1616-1627. 
37. Scholz J, Woolf CJ. The neuropathic pain triad: neurons, immune cells and glia. Nat Neurosci 2007;10:1361-1368.

38. Johnston IN, Milligan ED, Wieseler-Frank J, et al. A role for proinflammatory cytokines and fractalkine in analgesia, tolerance, and subsequent pain facilitation induced by chronic intrathecal morphine. J Neurosci 2004;24:7353-7365.

39. Milligan E, Zapata V, Schoeniger D, et al. An initial investigation of spinal mechanisms underlying pain enhancement induced by fractalkine, a neuronally released chemokine. Eur J Neurosci 2005;22:2775-2782.

40. Milligan ED, Zapata V, Chacur M, et al. Evidence that exogenous and endogenous fractalkine can induce spinal nociceptive facilitation in rats. Eur J Neurosci 2004;20:2294-2302.

41. Owolabi SA, Saab CY. Fractalkine and minocycline alter neuronal activity in the spinal cord dorsal horn. FEBS Lett 2006;580:4306-4310.

42. Johnston IN, Milligan ED, Wieseler-Frank J, et al. A role for proinflammatory cytokines and fractalkine in analgesia, tolerance, and subsequent pain facilitation induced by chronic intrathecal morphine. J Neurosci 2004;24:7353-7365.

43. Perkins NM, Tracey DJ. Hyperalgesia due to nerve injury: role of neutrophils. Neuroscience 2000;101:745-757.

44. Ramer MS, Murphy PG, Richardson PM, et al. Spinal nerve lesioninduced mechanoallodynia and adrenergic sprouting in sensory ganglia are attenuated in interleukin- 6 knockout mice. Pain 1998;78:115-121.

45. Zelenka M, Schafers M, Sommer C. Intraneural injection of interleukin1 beta and tumor necrosis factor-alpha into rat sciatic nerve at physiological doses induces signs of neuropathic pain. Pain 2005;116:257-263.

46. Gazda LS, Milligan ED, Hansen MK, et al. Sciatic inflammatory neuritis (SIN): behavioral allodynia is paralleled by peri-sciatic proinflammatory cytokine and superoxide production. J Peripher Nerv Syst 2001;6: 111-129.

47. Abbadie C. Chemokines, chemokine receptors and pain. Trends Immunol 2005:26:529-534.
48. Michalski CW, Selvaggi $F$, Bartel $M$, et al. Altered anti-inflammatory response of mononuclear cells to neuropeptide PACAP is associated with deregulation of NF-\{kappa\}B in chronic pancreatitis. Am J Physiol Gastrointest Liver Physiol 2008;294:G50-G57.

49. Milligan ED, Sloane EM, Watkins LR. Glia in pathological pain: a role for fractalkine. J Neuroimmunol 2008;198:113-120.

50. Perrin $\mathrm{FE}$, Lacroix $\mathrm{S}$, viles-Trigueros $\mathrm{M}$, et al. Involvement of monocyte chemoattractant protein-1, macrophage inflammatory protein-1alpha and interleukin-1beta in Wallerian degeneration. Brain 2005;128: 854-866.

51. Ito T. Can measurement of chemokines become useful biological and functional markers of early-stage chronic pancreatitis? J Gastroenterol 2007;42(Suppl 17):72-77.

52. Zhao HF, Ito T, Gibo J, et al. Anti-monocyte chemoattractant protein 1 gene therapy attenuates experimental chronic pancreatitis induced by dibutyltin dichloride in rats. Gut 2005;54:1759-1767.

53. Schneider E, Schmid-Kotsas A, Zhao J, et al. Identification of mediators stimulating proliferation and matrix synthesis of rat pancreatic stellate cells. Am J Physiol Cell Physiol 2001;281:C532-C543.

54. Saurer $L$, Reber $P$, Schaffner $T$, et al. Differential expression of chemokines in normal pancreas and in chronic pancreatitis. Gastroenterology 2000;118:356-367.

55. Furuichi K, Gao JL, Murphy PM. Chemokine receptor CX3CR1 regulates renal interstitial fibrosis after ischemia-reperfusion injury. Am J Pathol 2006;169:372-387.

56. Ishida Y, Gao JL, Murphy PM. Chemokine receptor CX3CR1 mediates skin wound healing by promoting macrophage and fibroblast accumulation and function. J Immunol 2008;180:569-579.

57. Imamura M, Ogawa $T$, Sasaguri $Y$, et al. Suppression of macrophage infiltration inhibits activation of hepatic stellate cells and liver fibrogenesis in rats. Gastroenterology 2005;128:138-146.

58. Ceyhan GO, Bergmann F, Kadihasanoglu M, et al. Pancreatic neuropathy and neuropathic pain-a comprehensive pathomorphological study in 546 cases. Gastroenterology 2008; doi: 10.1053/j.gastro.2008.09.029; 25 September 2008; e-pub ahead of print. 